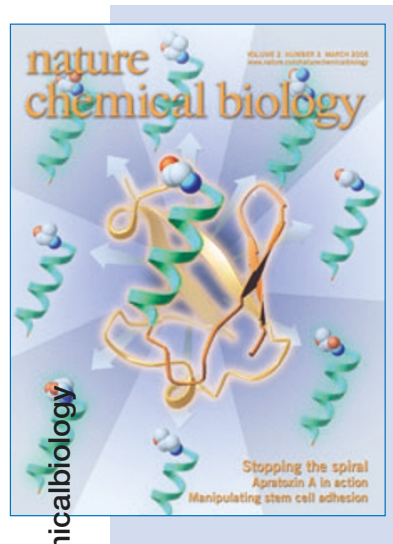

\section{Cover story}

The $\mathrm{C}$-terminal end of an $\alpha$-helix is terminated by a C-cap motif, which typically has glycine at the first position outside the helix. Glycine has a number of features unique among amino acids, including its small size and its ability to adopt a left-handed conformation. The energetic preference for glycine in the C-cap could result either from the optimal backbone solvation provided by the absence of a side chain or from the need for a left-handed conformation at this position. To dissect these potential explanations, Makhatadze,

Kent and colleagues used chemical protein synthesis to introduce $\mathrm{D}$-amino acids into the glycine $\mathrm{C}$-cap position. A thermodynamic comparison of the stabilities of proteins containing D-amino acids, which can favorably adopt a left-handed conformation, with proteins containing the corresponding L-amino acids led to the conclusion that adoption of a left-handed conformation makes glycine favored in the C-cap. [Letters, p. 139; News \& Views, p. 123]

$J K$

\section{Carbohydrates, quickly}

Bacteria display carbohydrates on their surface within lipopolysaccharides, polysaccharide capsules and glycoproteins. The composition of these carbohydrates changes rapidly in response to environmental stimuli. Mahal and coworkers have developed a lectin microarray, in which each lectin has a distinct carbohydrate-binding specificity. Fluorescently labeled bacteria bound to the array, revealing a glycan fingerprint that could be used to distinguish different bacterial strains. Because the microarray analysis was faster than any previous technique, the authors were able to monitor surface sugar changes during growth of a pathogenic strain of E. coli. This method provides an important tool for assessing dynamic changes in bacterial carbohydrates. [Letters, p. 153; News \& Views, p. 125]

$J K$

\section{Remodeled sugars change cell fate}

Thiol groups have diverse reactivities including nucleophilic attack and oxidation to form disulfide bonds. To determine the effects of extra thiols in cell surface sugars, Sampathkumar, Yarema and coworkers first modified the sialic acid precursor $\mathrm{N}$-acetyld-mannosamine (ManNAc) with a thiol derivative and fed it to cells. This allowed

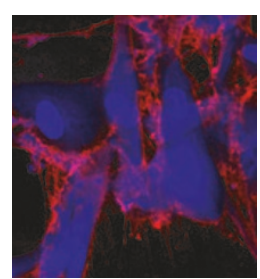

them to usurp the secretory pathway to deliver newly synthesized thiol-modified glycolipids or glycoproteins to the cell surface. In Jurkat T cells, this caused an obvious cell clustering phenotype, possibly owing to the fact that many disulfide bonds formed between the new thiols at the cell surface-sometimes with modified sialic acids from neighboring cells. In addition, anchorage to the extracellular matrix was strengthened. Human embryoid body-derived stem cells did not cluster but instead underwent progressive changes in gene expression and morphology characteristic of neuronal differentiation. Both types of changes were found to involve $\beta$-catenin, which is known to have a key role in both adhesion and signaling.

[Letters, p. 149; News \& Views, p. 127]

$M B$

In This Issue written by Mirella Bucci and Joanne Kotz.

\section{Apratoxin $\mathrm{A}$ in action}

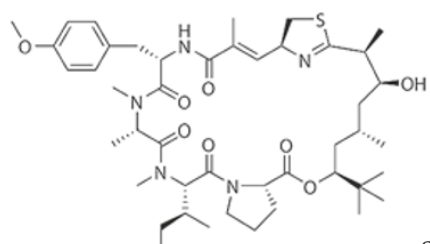

Apratoxin A, a natural product isolated from marine cyanobacteria, has been shown to have antitumor activity against a number of cancer cell lines. However, its mechanism of action is not known. Schultz, Luesch and coworkers found that apratoxin A induced cell-cycle arrest and apoptosis in cells. Using functional genomics approaches including a genome-wide overexpression screen and mRNA expression profiles, the authors found that increased fibroblast growth factor receptor (FGFR) signaling could reduce the effects of the small molecule. Apratoxin A was then found to inhibit a key phosphorylation of STAT3, a downstream effector of FGFR signaling. In agreement with these results, apratoxin A inhibited FGF signaling in vitro and in vivo. STAT3 is known to be activated in a number of cancers, so apratoxin A may provide a therapeutic lead. [Articles, p. 158]

$J K$

\section{Complete degradation}

Uric acid is produced from the breakdown of purines and is then further broken down to compounds that can be excreted. In most organisms, urate oxidase was believed to be the only enzyme involved in uric acid breakdown. However, the final product of the urate oxidase reaction is a racemic mixture of allantoin, whereas physiologically only $(S)$-allantoin is produced. Capitalizing on the fact that uric acid degradation has been lost multiple times during evolution-including, notably, in humans-Percudani and colleagues used a bioinformatics approach to search for additional proteins involved in uric acid degradation. The authors identified two proteins that, in combination with urate oxidase, resulted in the enzymatic conversion of uric acid to $(S)$-allantoin, thus completing the uric acid degradation pathway. [Letters, p. 144; News \& Views, p. 124]

$J K$

\section{Microbial iron wars}

To obtain the iron needed for their metabolism, enteric bacteria produce siderophores, highaffinity iron scavengers. Enterobactin is among the best-characterized catechol siderophores. In a Perspective article, Liu, Walsh and colleagues examine this

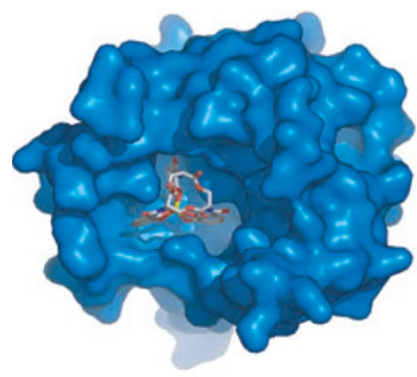
system in the context of bacterial cell invasion of their hosts. In their own struggle to maintain iron levels, mammalian cells harbor their own set of iron scavengers as well as an innate immunity response producing proteins such as siderocalin that can sequester enterobactin. Not to be outmatched, bacteria can use the iro $A$ gene cluster to generate enterocalin-modifying enzymes. This additional layer of secondary metabolism forms salmochelins, less hydrophobic versions of enterobactins that go undetected by mammalian cells. Yet a third metabolic tier comes into play as a large toxin is formed and camouflaged to facilitate bacterial iron uptake. These various levels of complexity reflect the high stakes involved in this evolving tug of war.

[Perspective, p. 132] 